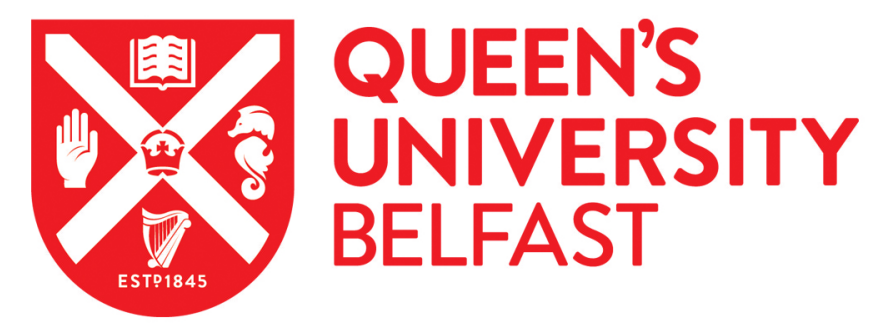

\title{
Multi-modal object detection using unsupervised transfer learning and adaptation techniques
}

\author{
Abbott, R., Robertson, N., Martinez del Rincon, J., \& Connor, B. (Accepted/In press). Multi-modal object \\ detection using unsupervised transfer learning and adaptation techniques. Paper presented at SPIE \\ Security+Defence, Strasbourg, France.
}

Document Version:

Early version, also known as pre-print

Queen's University Belfast - Research Portal:

Link to publication record in Queen's University Belfast Research Portal

Publisher rights

Copyright $\odot 2019$ SPIE. This work is made available online in accordance with the publisher's policies. Please refer to any applicable terms of use of the publisher.

\section{General rights}

Copyright for the publications made accessible via the Queen's University Belfast Research Portal is retained by the author(s) and / or other copyright owners and it is a condition of accessing these publications that users recognise and abide by the legal requirements associated with these rights.

Take down policy

The Research Portal is Queen's institutional repository that provides access to Queen's research output. Every effort has been made to ensure that content in the Research Portal does not infringe any person's rights, or applicable UK laws. If you discover content in the Research Portal that you believe breaches copyright or violates any law, please contact openaccess@qub.ac.uk. 


\title{
Multi-modal object detection using unsupervised transfer learning and adaptation techniques
}

\author{
Rachael Abbott ${ }^{\mathrm{a}}$, Neil Robertson ${ }^{\mathrm{a}}$, Jesus Martinez del Rincon ${ }^{\mathrm{a}}$, and Barry Connor ${ }^{\mathrm{b}}$ \\ aQueen's University Belfast \\ bThales UK
}

\begin{abstract}
Deep neural networks achieve state-of-the-art performance on object detection tasks with RGB data. However, there are many advantages of detection using multi-modal imagery for defence and security operations. For example, the IR modality offers persistent surveillance and is essential in poor lighting conditions and $24 \mathrm{hr}$ operation. It is, therefore, crucial to create an object detection system which can use IR imagery. Collecting and labelling large volumes of thermal imagery is incredibly expensive and time-consuming. Consequently, we propose to mobilise labelled RGB data to achieve detection in the IR modality. In this paper, we present a method for multi-modal object detection using unsupervised transfer learning and adaptation techniques. We train faster RCNN on RGB imagery and test with a thermal imager. The images contain object classes; people and land vehicles and represent real-life scenes which include clutter and occlusions. We improve the baseline F1-score by up to $20 \%$ through training with an additional loss function, which reduces the difference between RGB and IR feature maps. This work shows that unsupervised modality adaptation is possible, and we have the opportunity to maximise the use of labelled RGB imagery for detection in multiple modalities. The novelty of this work includes; the use of the IR imagery, modality adaption from RGB to IR for object detection and the ability to use real-life imagery in uncontrolled environments. The practical impact of this work to the defence and security community is an increase in performance and the saving of time and money in data collection and annotation.
\end{abstract}

Keywords: Object detection, Transfer learning, Modality adaption, Thermal imagery, Multi-modal detection

\section{INTRODUCTION}

The security and defence industry is becoming increasingly reliant on intelligent signal processing techniques to achieve $24 \mathrm{hr}$ surveillance capabilities. Machine learning can significantly benefit defence and security applications, for example, detecting threats in real-time can be used to support decision making. Automatic processing of the data is being implemented to reduce complexity and to fuse information from multiple sensors to increase reliability. By developing computer vision with $24 \mathrm{hr}$ surveillance, we can reduce the burden on the human operator, while allowing them to make safer, quicker and more informed decisions.

At present, object detection is predominantly applied to RGB imagery ${ }^{1-3}$, which exhibits severe limitations under low light or adverse weather conditions. However, detection in these situations is essential for defence and security operations. Infrared (IR) sensors can offer a solution to this problem. The current state of the art detectors (based on Convolutional neural networks (CNNs)) trained with RGB imagery have demonstrated very competitive performance. ${ }^{1}$ The main factor of this performance is the existence of extensive and well-annotated RGB datasets such as ImageNet, ${ }^{4} \mathrm{COCO}^{5}$ and Pascal VOC. ${ }^{2}$ However, there is currently a severe lack of publicly available IR data that prevents the development of effective detectors in IR imagery. Given the heavy dependency of CNNs on data, using small datasets for training can cause overfitting, and the model will fail to generalise. Collecting, processing, and annotating hundreds of thousands of IR images for deep learning algorithms would be prohibitively expensive or even impossible, given the context of this defence and security application.

Even with large datasets available for training, standard detectors cannot deal well with changes in data distributions including viewpoints, poses, lighting conditions, backgrounds and occlusions. Transfer Learning

Further author information: Send correspondence to rabbott02@qub.ac.uk 

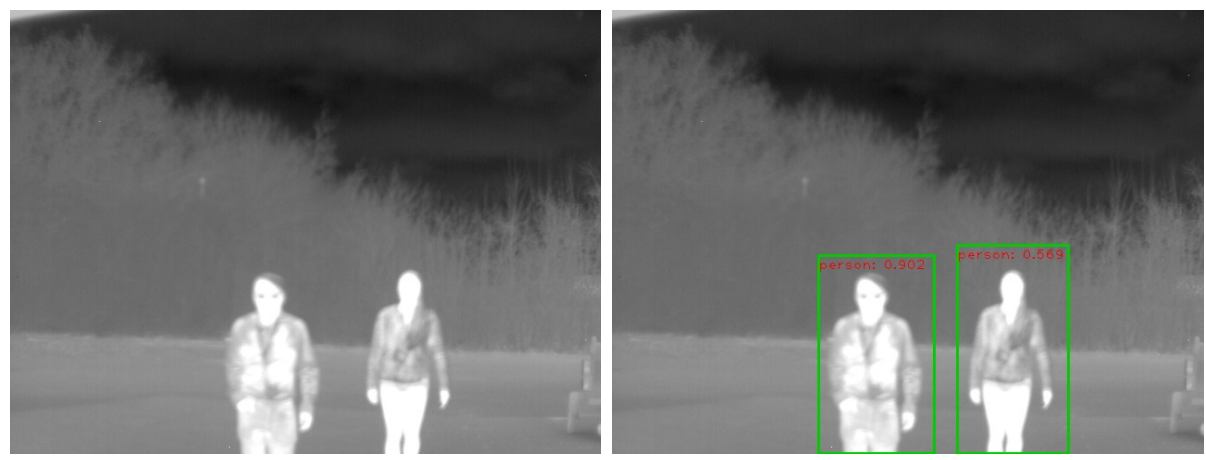

Figure 1. Detections in long wavelength IR imagery after training faster RCNN with RGB data with (left image) and without (right image) our extra mean squared error loss term.

(TL) and Domain Adaptation (DA) techniques help account for these changes in distributions. TL is the research area in machine learning that focuses on using the knowledge gained while solving one problem (source) and applying it to a different but related problem (target $)^{6}$. DA arises when we want to adapt a model trained on source data to perform well on a different but related target distribution. In the past number of years, many TL and DA techniques have been developed to help solve the lack of data issues in RGB imagery ${ }^{7}$. Our problem deals with changes in modality with the domain staying the same. To solve this problem of modality adaptation we ask two questions: can RGB trained weights be used for detection in IR imagery and is it possible to adapt a network trained on RGB imagery to boost the performance when tested with IR imagery in an unsupervised manner?

Our work addresses these questions and proposes an unsupervised modality adaptation approach to boost IR object detection performance. We train a network on labelled RGB and unlabelled IR imagery. During training, we aim to minimise the distance between the source (RGB) and target (IR) distributions as this will result in features which are invariant to shifts in modality. Figure 1 shows the effect of our method on LWIR imagery. To the best of our knowledge, this will be the first work addressing adaptation across modalities (RGB to IR) for object detection. The main contributions of our work are:

- Empirical proof that features learned from a network trained solely on RGB imagery can detect objects in IR imagery up to a certain degree of accuracy.

- Introduction of the concept of modality adaptation to enhance detection in IR imagery in an unsupervised manner.

- The first multi-modal detection network trained with RGB labels only.

- A demonstration that our method works for non-corresponding imagery which is ultimately more valuable for real-life scenarios.

\section{RELATED WORK}

Given that we are introducing the concept of modality adaptation between RGB and IR imagery we, therefore, describe relevant research on TL and DA with a focus on deep learning methods.

TL is usually followed by fine-tuning (FT) on the target specific dataset if labels are provided (supervised domain adaptation). The majority of research which incorporate these techniques use RGB data ${ }^{8}$. However, recently TL techniques have explored fusing RGB and thermal ${ }^{9,10}$ and thermal/RGB and depth imagery to improve detection results ${ }^{11,12}$. TL alone is not sufficient for solving our problem as not all features will be useful for the target imagery. More importantly, FT requires target labels, which is not always available, like our scenario in this paper. There are several unsupervised adaptation techniques available when TL followed by FT is not possible. Many unsupervised DA techniques learn correspondences in features between two different 
domains by minimising the difference between the source and target feature distributions. Several researchers have used maximum mean discrepancy ${ }^{13}$ loss for this purpose ${ }^{14,15}$. In 2014, Ganin and Lempitsky ${ }^{16}$ presented a new approach to domain adaptation using a domain classifier. The network is invariant to shifts in the domain by augmenting it with a gradient reversal layer. Through training, the domain classifier finds it harder to distinguish between domains as the features learnt become more invariant to changes in domain. In papers, ${ }^{17-20}$ similar routes are taken. The authors of paper $^{21}$ use multiple adversarial discriminators to make use of complex structures of the target domain and improve performance. Some methods ${ }^{22,23}$ aim to mitigate this issue by using pseudo labels for training in the target domain in a semi-supervised approach. Pseudo labels are the predicted class labels of the object with the maximum probability, used as if they are correct. Results can be unstable when there are few labelled examples ${ }^{23}$. Researchers have more recently started to use generative adversarial networks (GANs) to make source imagery look as though it came from the target domain ${ }^{24-27}$. Object detection in defence and security in computer vision is rarely covered, with only a small number of papers applying current techniques to military data compared with other applications ${ }^{28-30}$. Military data is very complex, and this may be a reason why research rarely uses it.

These adaptation techniques have not been tested for modality adaptation. In our work, we address this by using an adaptation technique which minimises the mean squared error between feature maps in RGB and IR imagery. We train without requiring corresponding imagery in both modalities. We choose not to train with pseudo labels as our dataset was small and the shift between modalities is more significant than between domain so falsely labelled data would greatly affect our network. GANs were not used to create synthetic IR imagery as my dataset contains complex scenes and it is impossible to determine heat signatures from RGB imagery. We use the mean squared error as it is a simple but effective way to measure the difference between feature maps and help create modality invariant features.

\section{OUR APPROACH}

Our object detection network is trained to generate features which are invariant to changes in modality. To create these features, we introduce an additional loss function into the Faster RCNN (fRCNN) network ${ }^{1}$ losses. $^{2}$ We use this loss to help train the network to minimise the difference between the feature maps produced from both RGB and long-wave infrared (LWIR) imagery. Note that we do not use LWIR labels in the training process and so it can be considered as unsupervised adaptation. Figure 2 depicts the full architecture of our proposed network.

\subsection{Object detection network}

We use the popular fRCNN network with VGG16 architecture ${ }^{31}$ as the baseline feature extractor. This network has a two-stage detection approach: localisation and classification. Object localisation is the process of finding where objects are in images and creating a bounding box around them. Object classification will provide a label identifying each of the objects in the bounding boxes.

\subsection{Faster RCNN loss}

Within the fRCNN architecture, the region proposal network (RPN) produces a confidence probability to show how confident the net is that there is an object present in the current anchor box. Anchor boxes are a set of predefined bounding boxes of a certain height and width. The RPN also creates anchor box regression values to predict where the network believes there are objects present. The final evaluation layers of the fRCNN network predict probabilities for classification and bounding box information. Therefore, the fRCNN loss consists of a region proposal objectness loss, a region proposal anchor loss, a classification loss and a bounding box loss ${ }^{1}$. Equations $1-4$ express the components of the loss mathematically.

The anchor loss $(A)$ is given as:

$$
A=\lambda \frac{1}{N_{A}} \sum_{i} p_{i}^{*} L_{r e g}\left(t_{i}, t_{i}^{*}\right)
$$

where $L_{r e g}$ is the smooth L1 loss function, $t_{i}$ represents the vector containing the coordinates of the proposal anchor box, $t_{i}^{*}$ is the ground truth box associated with a positive anchor box. $N_{A}$ is the total number of anchor 


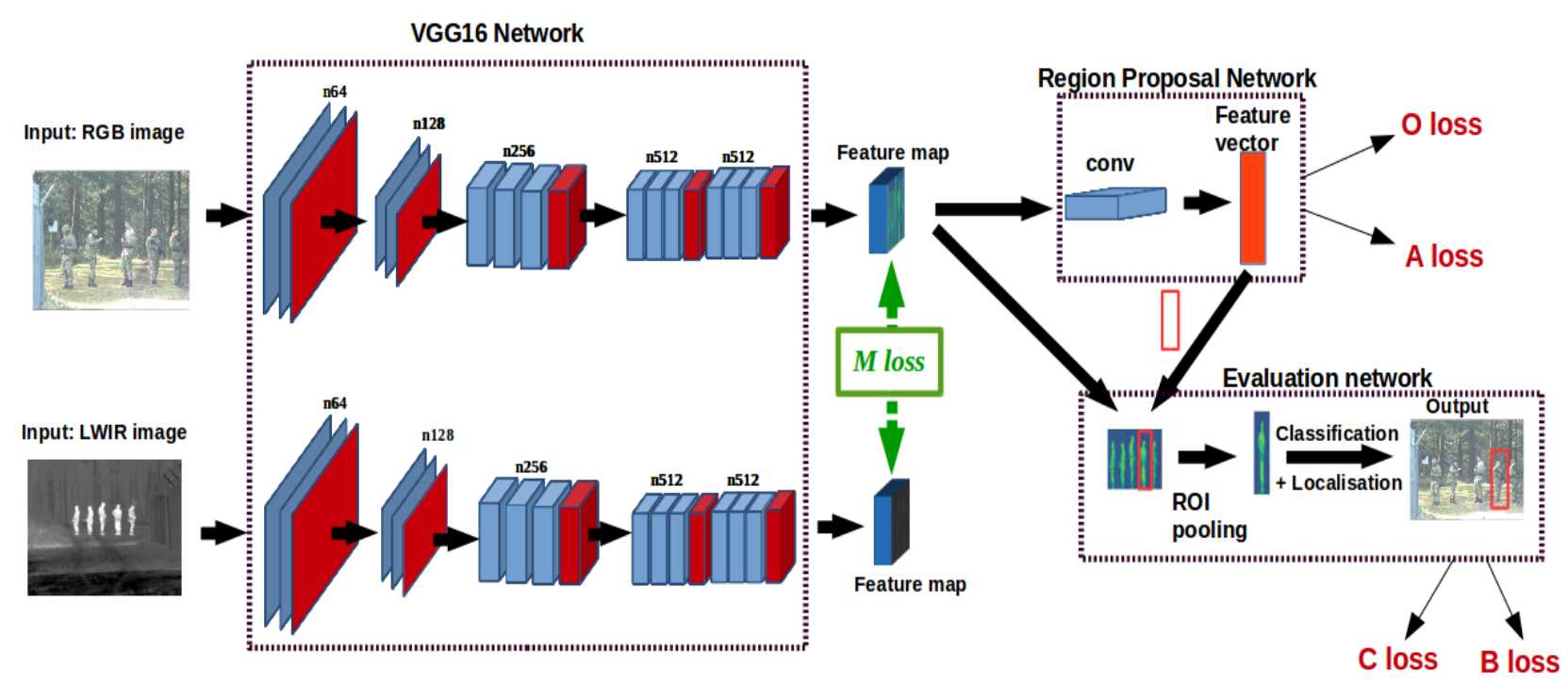

Figure 2. Diagram depicting the fRCNN with VGG16 architecture that the RGB imagery passes through and our proposed extension to adapt the network to LWIR imagery. The feature extractor of the fRCNN network consists of the first 13 convolutional layers of the VGG16 network. After every convolution (blue), there is a RELU layer and every second/third layer there is a max pooling layer (red).

boxes and $\lambda$ is a balancing weight and has a default value of ten ${ }^{1} \cdot p_{i}$ is the predicted probability that the anchor box contains an object. The ground truth probabilities $p_{i}^{*}$ are 1 if the anchor contains an object and 0 if it does not. $N$ is the number of anchor boxes. Note that only positively labeled anchors are used for training.

The objectness loss $(O)$ is described as:

$$
O=\frac{1}{N_{c l s}} \sum_{i=1}^{N} L_{c l s}\left(p_{i}, p_{i}^{*}\right)
$$

where $L_{c l s}$ is the log loss over two class objects i.e. object present versus no object present. This loss is normalised by $N_{c l s}$ which is the mini-batch size that the network is trained with.

For each region of interest (ROI), there are bounding box regression offsets $B^{k}=\left(B_{x}^{k}, B_{y}^{k}, B_{w}^{k}, B_{h}^{k}\right)$ for each of the $\mathrm{K}$ object classes, indexed by $\mathrm{k}$ and discrete probability outputs $\operatorname{prob}=\left(\right.$ prob $_{0}, \ldots$, prob $\left._{K}\right)$. Each training ROI is labelled with a ground truth class $u$ and bounding box regression $v$.

The Bounding Box loss $(B)$ and Classification loss $(C)$ are described as:

$$
\begin{gathered}
B=[u \geq 1] L_{l o c}\left(B^{u}, v\right) \\
C=-\log \left(\operatorname{prob}_{u}\right)
\end{gathered}
$$

where $L_{l o c}$ is the smooth L1 loss function.

\subsection{Mean Squared Error (MSE) Loss}

RGB and LWIR images input into the fRCNN network trained only on RGB imagery, will produce very different feature maps. Therefore, detection in the LWIR imagery will be a lot lower than for RGB. As we have only labels for the RGB imagery, the LWIR image cannot go through the RPN or evaluation network, as shown in Figure 2. Our solution is to make the RGB and IR feature maps produced from the VGG16 network as similar as possible. 


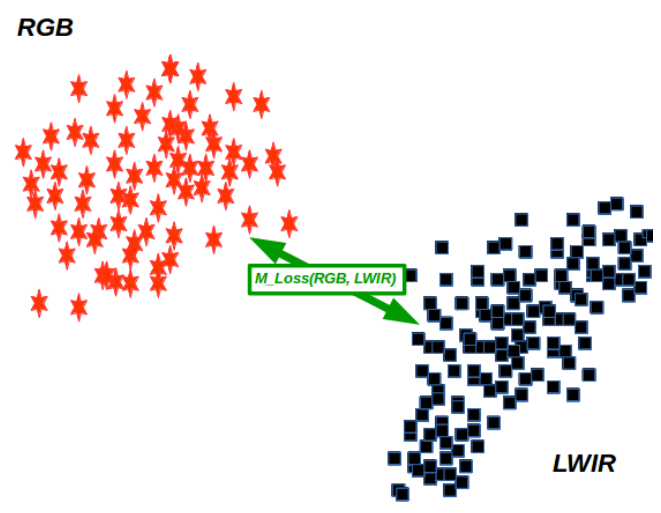

Figure 3. 2D feature space representation of our approach to modality adaptation. The figure shows the RGB and LWIR sub classes inside the modality class feature space. We use the $M_{\text {loss }}$ to decrease the distance between the two modality sub classes as shown by the green arrow.

To do this, we minimise the difference between the feature maps (of RGB and LWIR imagery) and add this to the loss function to update the network during training. Figure 3 shows the 2D feature space representation of this problem.

Among the multiple ways to measure the difference between the two feature maps, we use the mean squared error (MSE) for our task as it is a simple but effective way to measure the difference between feature maps. The MSE loss is shown in the following equation:

$$
M_{\text {loss }}=\frac{1}{r * t} \sum_{l=1}^{s} \sum_{m=1}^{r} \sum_{n=1}^{t}\left(X_{m, n}^{l}-Y_{m, n}^{l}\right)^{2}
$$

where $r * t$ are the total number of values in one feature map, $s$ is the total number of feature maps, $X_{r, t}$ is a value at point $r, t$ in a feature map produced from an RGB image and $Y_{r, t}$ is a value in point $r, t$ of the feature map produced from a LWIR image. Figure 2 shows where we apply the $M_{\text {loss }}$ in the fRCNN network architecture when training.

Figure 2 shows how the RGB and LWIR image are passed through the same VGG16 network and produce feature maps. The MSE of these features maps is calculated and added to the loss function. The RGB feature map produced then passes into the region proposal network. This network creates proposals (i.e. bounding boxes) for suspected objects. These proposals combine with the feature map produced after extraction for region of interest pooling which produces a crop of a suspected object which is then classified. When training, the network is updated with the four fRCNN losses plus the MSE loss.

\subsection{Final loss}

The final loss is formulated as the sum of the fRCNN loss denoted as $F_{\text {loss }}$ and the Mean squared error loss $M_{\text {loss }}$ such that:

$$
\text { Final }_{\text {loss }}=F_{\text {loss }}+\alpha M_{\text {loss }}
$$

where $F_{\text {loss }}=A+O+B+C$ and $\alpha$ is a weighting factor to balance the influence of the modality adaptation during training. While perfect correspondence between RGB and IR will make the adaptation more successful, this schema can also work for non corresponding images, as we demonstrate in the experimental section. The value of $\alpha$ can be tuned according to this and is determined experimentally as shown in the results section.

\section{EXPERIMENTS}

This section describes the two datasets used in our experimentation to validate our approach and the results produced. 

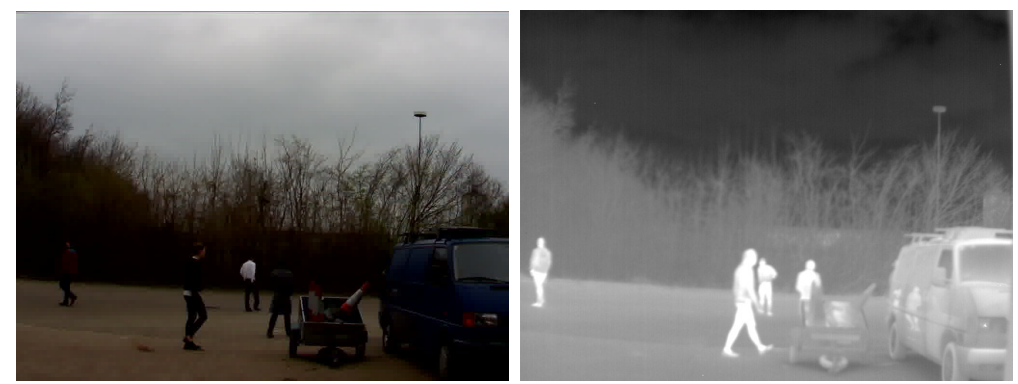

Figure 4. Examples of imagery in Thales dataset from the RGB and LWIR cameras. Images are rescaled for display purposes.

\subsection{Datasets}

Two RGB-LWIR datasets are used to validate our approach: the FLIR publicly available dataset ${ }^{32}$ and a dataset made using a Thales thermal camera * and a commercially available RGB camera.

\subsubsection{Thales Dataset}

We create our RGB-LWIR dataset using military grade defence cameras and capture unsynchronised (cameras did not start and end at the same time) RGB and LWIR imagery. Cameras were mounted next to each other on a tripod so the fields of view are similar but not identical. The RGB camera has a resolution of $1024 \times 768$ and Thales LWIR imager has a resolution of $640 \times 480$. Weather in the imagery is cloudy, dull and dry and the background consists of the sky, trees, bushes and a paved light coloured road. There are a stationary vehicle and trailer to the left in all the imagery. The number of people in the imagery range from one to five. They travel on random walks and are regularly occluded by each other, the vehicle, the trailer, and when they walk out of the field of view of the camera. There are instances of people lunging, crouching, making irregular shapes with their bodies and hiding behind the trailer and vehicle. Object classes included in labelling are people and land vehicle. The dataset contains 738 labelled images. Examples from this dataset are given in Figure 4.

\subsubsection{FLIR dataset}

The FLIR dataset consists of synced (in time) annotated thermal imagery (640 x 512) and non-annotated RGB imagery (1800 x 1600) taken in Santa Barbara, California. Cameras were approximately $5 \mathrm{~cm}$ apart and collimated to minimize parallax. The RGB imagery has a larger field of view than the LWIR imagery; the field of view for the LWIR imagery is contained within the RGB field of view. Therefore, to use the LWIR annotations provided for the RGB imagery, some preprocessing of the RGB imagery was required. 1252 labelled images were used in our experiments.

\subsubsection{Experimental setting}

Our experiments use pre-trained ImageNet models for our VGG16 network that are available online. We use cross validation to evaluate our networks using $70 \%$ of the images for training and $30 \%$ for testing. Of the available classes we use both vehicle and people. After investigating the effect of different alpha values on our network, we choose alpha to be $10^{-4}$ empirically for the Thales dataset (Figure 5) and $10^{-6}$ for the FLIR dataset. We use the F1 metric as it is the weighted average of Precision and Recall. This score takes both false positives and false negatives into account. The F1 score is usually more useful than accuracy, especially when there is an uneven class distribution and it is a widely used metric in machine learning. A detection is classified as correct if the predicted bounding box has overlap of $50 \%$ or more with the ground truth and is classified with the correct label.

\subsection{Results}

This subsection presents the F1 scores produced for the two datasets we use in our experimentation.

\footnotetext{
${ }^{*}$ Contact Thales UK for more detail
} 


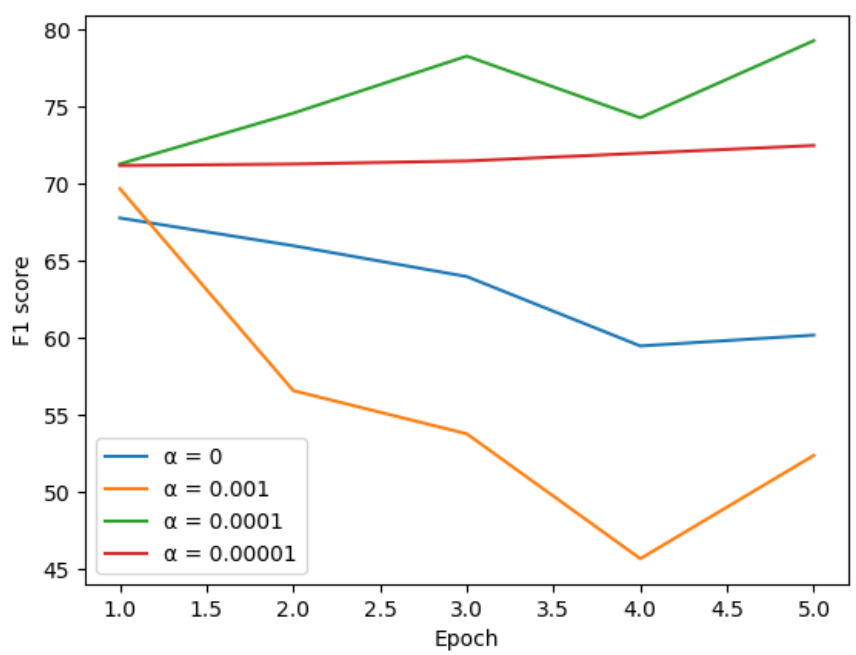

Figure 5. LWIR F1 scores with $\alpha$ when trained on Thales dataset.

\subsubsection{Thales dataset results}

Table 1 shows the F1 scores with and without adaptation on the Thales LWIR and RGB dataset for five epochs. By looking solely to the non-adapted version, it can be seen that F1 scores for the LWIR imagery decrease after every epoch when training on RGB data. This is because with every passing epoch, the features are becoming more specific to the RGB imagery. When using our proposed metric combining $M_{\text {loss }}$ and $F_{\text {loss }}$, the RGB F1 score more or less stays the same while the F1 score for LWIR improves with nearly every epoch. We choose to compare the F1 score from the fifth epoch as this is when training for the RGB maximises. The experiment was repeated three times to show that the MSE loss consistently improves the F1 scores on average by $20 \%$. We conclude that the network is not only adapting to the object detection task but also learning more modality invariant features with every epoch thanks to the use of our proposed DA.

Table 1. F1 scores for training with and without $M_{\text {loss }}$ for Thales dataset.

\begin{tabular}{|l|l|l|l|}
\hline Loss & epoch & RGB F1 & LWIR F1 \\
\hline$F_{\text {loss }}$ & 1 & 86.8 & 67.8 \\
\hline$F_{\text {loss }}$ & 2 & 87.1 & 66 \\
\hline$F_{\text {loss }}$ & 3 & 92.9 & 64 \\
\hline$F_{\text {loss }}$ & 4 & 93.8 & 59.5 \\
\hline$F_{\text {loss }}$ & 5 & 95.3 & $\mathbf{6 0 . 2}$ \\
\hline \hline$F_{\text {loss }}+10^{-4} * \mathrm{M}_{\text {loss }}$ & 1 & 79.3 & 71.3 \\
\hline$F_{\text {loss }}+10^{-4} * \mathrm{M}_{\text {loss }}$ & 2 & 88.5 & 74.6 \\
\hline$F_{\text {loss }}+10^{-4} * \mathrm{M}_{\text {loss }}$ & 3 & 91.9 & 78.3 \\
\hline$F_{\text {loss }}+10^{-4} * \mathrm{M}_{\text {loss }}$ & 4 & 91.6 & 74.3 \\
\hline$F_{\text {loss }}+10^{-4} * \mathrm{M}_{\text {loss }}$ & 5 & 93.8 & $\mathbf{7 9 . 3}$ \\
\hline
\end{tabular}

\subsubsection{FLIR dataset results}

Results using the FLIR dataset in Table 2 show that the MSE loss can increase the F1 score on LWIR imagery by $9 \%$ and $12 \%$ when using non-corresponding and corresponding RGB-LWIR imagery respectively. Corresponding 
imagery shows the biggest improvement in F1 scores, but our method still works otherwise. This is a great advantage in many situations where there are no simultaneous cameras mounted in the same locations/vehicle.

Table 2. F1 scores for training with and without $M_{\text {loss }}$ for FLIR dataset.

\begin{tabular}{|l|l|l|l|}
\hline Loss & epoch & RGB F1 & LWIR F1 \\
\hline$F_{\text {loss }}$ & 5 & 46.6 & 30.8 \\
\hline$F_{\text {loss }}$ & 10 & 64.5 & 29.7 \\
\hline$F_{\text {loss }}$ & 15 & 64.2 & $\mathbf{2 9 . 7}$ \\
\hline \hline$F_{\text {loss }}+10^{-6} * \mathrm{M}_{\text {loss }}($ non - corresponding data $)$ & 5 & 51.9 & 27.6 \\
\hline$F_{\text {loss }}+10^{-6} * \mathrm{M}_{\text {loss }}($ non - corresponding data $)$ & 10 & 64.3 & 32.0 \\
\hline$F_{\text {loss }}+10^{-6} * \mathrm{M}_{\text {loss }}($ non - corresponding data $)$ & 15 & 64.5 & $\mathbf{3 8 . 7}$ \\
\hline \hline$F_{\text {loss }}+10^{-6} * \mathrm{M}_{\text {loss }}($ corresponding data $)$ & 5 & 43.2 & 29.6 \\
\hline$F_{\text {loss }}+10^{-6} * \mathrm{M}_{\text {loss }}$ (corresponding data $)$ & 10 & 63.8 & 42.9 \\
\hline$F_{\text {loss }}+10^{-6} * \mathrm{M}_{\text {loss }}$ (corresponding data) & 15 & 63.9 & $\mathbf{4 2 . 3}$ \\
\hline
\end{tabular}

\section{DISCUSSION}

From the results Tables $(1,2)$, we can see that the MSE loss helps improve the detection rate in LWIR imagery. The FLIR dataset has a lower initial F1 score than the Thales dataset as this imagery is more complex and represents a more difficult detection problem. These images are incredibly cluttered, have changing backgrounds and object classes are continually occluded. Some scenes have ten plus objects classes per image with many objects overlapping. As a reference value, FLIR produces a mean average precision (mAP) of $58.7 \%$ after training and testing on the LWIR imagery using RefineDetect512 ${ }^{32}$. We use the FLIR dataset in our paper to show that transfer learning is possible even in cluttered scenes and not specific to Thales cameras. With this dataset, we also have the opportunity to show the importance of correspondence between the RGB and thermal imagery. Having corresponding (RGB/LWIR imagery lined up both in time and space) imagery maximises adaption process as can be seen in Table 2. This was expected given the definition of MSE loss in equation 5, where pixel by pixel comparison is performed. However, corresponding imagery is not always possible, so the fact that this method works in non-corresponding imagery means that it is more applicable to real-life situations.

\subsubsection{Learned feature space}

As shown in the schematic in Figure 6, training without MSE produces a feature space with the RGB and LWIR feature maps at a distance to each other. The MSE loss function influences the feature space, so changes in modalities are less pronounced, as shown in Figure 6 with real data. If the MSE loss is too large, it can create a feature space with the RGB and LWIR completely overlapping but this can cause detection rates to fall to zero. This is because the MSE loss overpowers the other losses and the network learns only to create modality invariant features and not how to detect and classify objects. Figure 6 represents the compromise between creating modality invariant features and learning to detect objects and classify them correctly.

\section{CONCLUSION}

In this paper, we proposed the use of transfer learning and adaptation techniques for multi-modal object detection in a security and defence context. We use labelled RGB data for training our network to help detect in the LWIR modality. Specifically, we propose the application of the mean squared error loss for unsupervised modality adaptation from RGB to LWIR imagery that doesn't require corresponding imagery between both modalities. Results in two different datasets show a promising $10-20 \%$ increase in F1 scores for LWIR images while maintaining their RGB performance. The defence and security industry can benefit from our work by achieving 24 hour detection which does not require time and money spent collecting, processing and annotating IR data. 

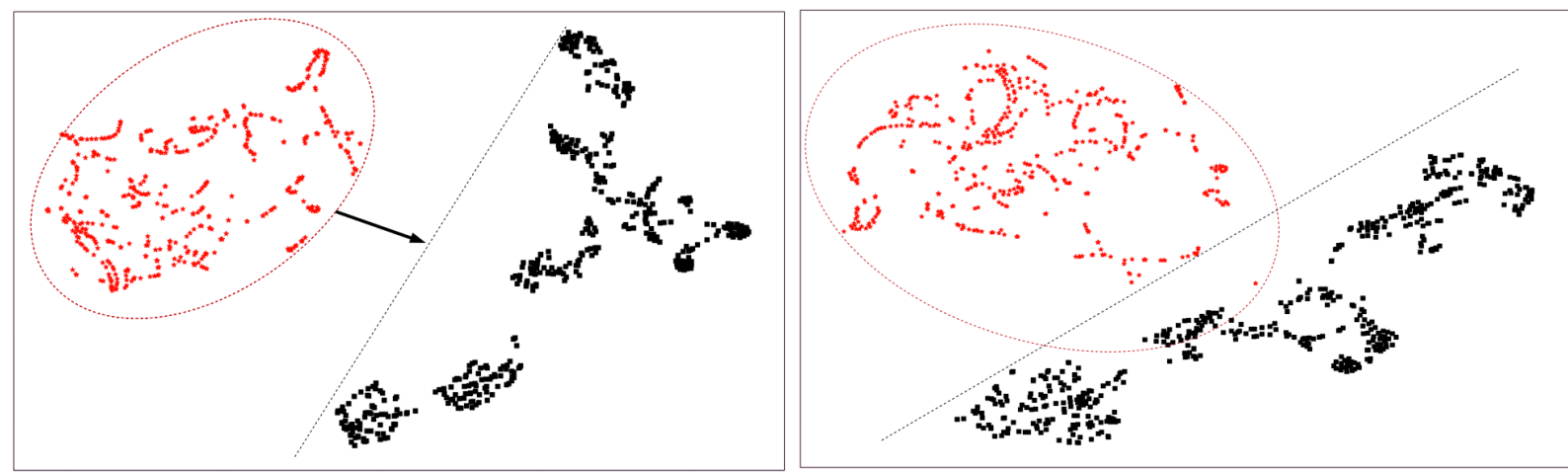

Figure 6. Representation of our approach to the optimization of the MSE loss and its overall effect on the feature space. Red stars and black squares represent the RGB and LWIR features respectively. The black arrow represents the direction we want the RGB features to move in.

\section{REFERENCES}

[1] Ren, S., He, K., Girshick, R. B., and Sun, J., "Faster R-CNN: towards real-time object detection with region proposal networks," CoRR abs/1506.01497 (2015).

[2] Everingham, M., Van Gool, L., Williams, C. K. I., Winn, J., and Zisserman, A., "The pascal visual object classes (voc) challenge," International Journal of Computer Vision 88, 303-338 (June 2010).

[3] Redmon, J. and Farhadi, A., "YOLO9000: better, faster, stronger," CoRR abs/1612.08242 (2016).

[4] Deng, J., Dong, W., Socher, R., Li, L.-J., Li, K., and Fei-Fei, L., "ImageNet: A Large-Scale Hierarchical Image Database," in [CVPR09], (2009).

[5] Lin, T.-Y., Maire, M., Belongie, S., Hays, J., Perona, P., Ramanan, D., Dollár, P., and Zitnick, C. L., "Microsoft coco: Common objects in context," in [Computer Vision - ECCV 2014], Fleet, D., Pajdla, T., Schiele, B., and Tuytelaars, T., eds., 740-755, Springer International Publishing, Cham (2014).

[6] Khan, S., Islam, N., Jan, Z., Din, I. U., and Rodrigues, J. J. P. C., "A novel deep learning based framework for the detection and classification of breast cancer using transfer learning," Pattern Recognition Letters 125, $1-6(2019)$.

[7] Donahue, J., Jia, Y., Vinyals, O., Hoffman, J., Zhang, N., Tzeng, E., and Darrell, T., "Decaf: A deep convolutional activation feature for generic visual recognition," CoRR abs/1310.1531 (2013).

[8] Razavian, A. S., Azizpour, H., Sullivan, J., and Carlsson, S., "CNN features off-the-shelf: an astounding baseline for recognition," CoRR abs/1403.6382 (2014).

[9] Bosilj, P., Aptoula, E., Duckett, T., and Cielniak, G., "Transfer learning between crop types for semantic segmentation of crops versus weeds in precision agriculture," Journal of Field Robotics $\mathbf{0}(0)$.

[10] Liu, S. and Liu, Z., "Multi-channel cnn-based object detection for enhanced situation awareness," CoRR abs/1712.00075 (2017).

[11] Hoffman, J., Gupta, S., Leong, J., Guadarrama, S., and Darrell, T., "Cross-modal adaptation for rgb-d detection," in [2016 IEEE International Conference on Robotics and Automation (ICRA)], 5032-5039 (May 2016).

[12] Chen, Z. and Huang, X., "Pedestrian detection for autonomous vehicle using multi-spectral cameras," IEEE Transactions on Intelligent Vehicles PP, 1-1 (03 2019).

[13] Gretton, A., Borgwardt, K. M., Rasch, M. J., Schölkopf, B., and Smola, A., "A kernel two-sample test," J. Mach. Learn. Res. 13, 723-773 (Mar. 2012).

[14] Ghifary, M., Kleijn, W. B., and Zhang, M., "Domain adaptive neural networks for object recognition," CoRR abs/1409.6041 (2014).

[15] Liu, L., Lin, W., Wu, L., Yu, Y., and Yang, M. Y., "Unsupervised deep domain adaptation for pedestrian detection," CoRR abs/1802.03269 (2018).

[16] Ganin, Y. and Lempitsky, V., "Unsupervised domain adaptation by backpropagation," in [Proceedings of the 32nd International Conference on Machine Learning], Bach, F. and Blei, D., eds., Proceedings of Machine Learning Research 37, 1180-1189, PMLR, Lille, France (07-09 Jul 2015). 
[17] Koga, Y., Miyazaki, H., and Shibasaki, R., "Deep domain adaptation for single shot vehicle detector in sateliite images," IEEE International Geoscience and remote sensing symposium $\mathbf{P P}$ (2018).

[18] Tzeng, E., Hoffman, J., Saenko, K., and Darrell, T., "Adversarial discriminative domain adaptation," CoRR abs/1702.05464 (2017).

[19] Tzeng, E., Hoffman, J., Saenko, K., and Darrell, T., "Adversarial discriminative domain adaptation," CoRR abs/1702.05464 (2017).

[20] Ye, F., Luo, W., Dong, M., He, H., and Min, W., "Sar image retrieval based on unsupervised domain adaptation and clustering," IEEE Geoscience and Remote Sensing Letters , 1-5 (2019).

[21] Pei, Z., Cao, Z., Long, M., and Wang, J., "Multi-adversarial domain adaptation," CoRR abs/1809.02176 (2018).

[22] Zou, Y., Yu, Z., Kumar, B. V. K. V., and Wang, J., "Domain adaptation for semantic segmentation via class-balanced self-training," CoRR abs/1810.07911 (2018).

[23] Xie, S., Zheng, Z., Chen, L., and Chen, C., "Learning semantic representations for unsupervised domain adaptation," in [Proceedings of the 35th International Conference on Machine Learning], Dy, J. and Krause, A., eds., Proceedings of Machine Learning Research 80, 5423-5432, PMLR, Stockholmsmssan, Stockholm Sweden (10-15 Jul 2018).

[24] Bousmalis, K., Silberman, N., Dohan, D., Erhan, D., and Krishnan, D., "Unsupervised pixel-level domain adaptation with generative adversarial networks," CoRR abs/1612.05424 (2016).

[25] Huang, X., Liu, M., Belongie, S. J., and Kautz, J., "Multimodal unsupervised image-to-image translation," CoRR abs/1804.04732 (2018).

[26] Deng, W., Zheng, L., Kang, G., Yang, Y., Ye, Q., and Jiao, J., "Image-image domain adaptation with preserved self-similarity and domain-dissimilarity for person re-identification," CoRR abs/1711.07027 (2017).

[27] Liu, M. and Tuzel, O., "Coupled generative adversarial networks," CoRR abs/1606.07536 (2016).

[28] Bhatnagar, G. and Liu, Z., "A novel image fusion framework for night-vision navigation and surveillance," Signal, Image and Video Processing 9, 165-175 (Dec 2015).

[29] Sheeny, M., Wallace, A., Emambakhsh, M., Wang, S., and Connor, B., "POL-LWIR vehicle detection: Convolutional neural networks meet polarised infrared sensors," CoRR abs/1804.02576 (2018).

[30] Abbott, R., Del Rincon, J., Connor, B., and Robertson, N., "Deep object classification in low resolution lwir imagery via transfer learning," in [Proceedings of the 5th IMA Conference on Mathematics in Defence], (11 2017).

[31] Simonyan, K. and Zisserman, A., "Very Deep Convolutional Networks for Large-Scale Image Recognition," arXiv e-prints, arXiv:1409.1556 (Sep 2014).

[32] F.A.Group, "Flir thermal dataset for algorithm training." https://www.flir.co.uk/oem/adas/ adas-dataset-form/. Accessed: 05-07-2019. 